\title{
Identification of two distinct genes at the vertebrate TRPC2 locus and their characterisation in a marsupial and a monotreme
}

\author{
Stephen Frankenberg ${ }^{\dagger}$, Nanette Y Schneider ${ }^{\dagger}$, Terrence P Fletcher, Geoffrey Shaw and Marilyn B Renfree*
}

\begin{abstract}
Background: The vomeronasal organ (VNO) detects pheromones via two large families of vomeronasal receptors: vomeronasal receptor 1 (V1R) and vomeronasal receptor 2 (V2R). Both VRs have a common receptor activation cascade involving transient receptor potential channel, subfamily C, member 2 (TRPC2).

Results: We characterised the TRPC2 locus in a marsupial, the tammar wallaby (Macropus eugenii), and identified two independently regulated genes not previously recognised as distinct. 3'-located exons comprise bona fide TRPC2 whilst 5'-located exons, previously identified as part of TRPC2, comprise a distinct gene, which we term XNDR (XRCC1 N-terminal domain-related). The two genes show contrasting expression patterns in the tammar: TRPC2 is specifically expressed in adult and developing VNO, whereas XNDR is widely expressed in many tissues suggesting a non-VNO-specific role. Strong expression of TRPC2 was detected only after about day 30 post-partum, suggesting that the VNO may not be functional during early pouch life of the tammar. Similarly restricted expression of TRPC2 and widespread expression of XNDR was also detected in the platypus. Bioinformatic analysis of the genomes of a wide range of species suggests that the identity of XNDR and TRPC2 as distinct genes is conserved among vertebrates. Finally, we analysed the promoter of mammalian TRPC2 and identified a conserved binding site for $\mathrm{NHLH1}$, a transcription factor previously implicated in $\mathrm{VNO}$ receptor neuron development.
\end{abstract}

Conclusions: Two functionally distinct vertebrate genes-XNDR and TRPC2 - occupy a genomic locus that was previously defined as a single gene in the mouse. The former is widely expressed with a putative role in DNA repair, while the latter shows VNO-specific expression under the probable regulation of $\mathrm{NHLH}$.

\section{Background}

The vomeronasal organ (VNO) is a paired tubular organ that is found in the nasal cavity of most tetrapods [1,2]. It lies in the tissue on either side of the nasal septum, stretching along its sides towards the back of the nasal cavity. The VNO is thought mainly to detect pheromones or pheromone blends [3], but it also detects some environmental odors $[4,5]$. Functioning of the VNO requires functional receptor cells with connections to the brain and all parts of the receptor activation cascade. Two families of vomeronasal receptors (VRs)-V1Rs and V2Rs-are specific to the $\mathrm{VNO}$ and the number of family members varies greatly between species (e.g. [6-10], making comparative

\footnotetext{
* Correspondence: m.renfree@zoology.unimelb.edu.au

+ Contributed equally

Department of Zoology, The University of Melbourne, Melbourne, Victoria, 3010, Australia
}

studies difficult. Not all mammals have intact receptors of both families. The dog (Canis familiaris) and the cow (Bos taurus), for example, only have intact $V 1 R$ genes and lack functional $V 2 R$ genes [8,9]. Both vomeronasal receptors are thought to use a common receptor activation cascade that depends on the transient receptor potential channel protein, subfamily C, member 2 (TRPC2) [11,12]. TRPC2 is one of seven known TRPCs [13]. While the activation process is still not fully understood, the TRPC2 channel is thought to be modulated through phospholipase C [14]. $T R P C 2$ represents its own gene subfamily as it is quite dissimilar to the other TRPCs in sequence and function [15]. To date, full-length transcripts of TRPC2 have been described for the mouse (Mus musculus) [16], rat (Rattus norvegicus) [12], New World monkeys [17-19], cow [20], Californian sea lion (Zalophus californianus) [21] and zebrafish (Danio rerio) [22], whereas TRPC2 in human (Homo

\section{() Biomed Central}


sapiens) [23], Old World monkeys [17,19], dolphin (Tursiops truncatus), little brown bat (Myotis lucifugus), flying fox (Pteropus vampyrus) [10], fin whale (Balaenoptera physalus), harbour seal (Phoca vitulina) and river otter (Lutra lutra) [21] contains stop codons, indicating a disabled pseudogene. Partial sequences of TRPC2 have been characterised for the elephant shark (Callorhinchus milii) and sea lamprey (Petromyzon marinus) but not in nonvertebrate chordates such as lancelet (Branchiostoma floridae) and sea squirt (Ciona intestinalis), suggesting that TRPC2 was already present in the olfactory epithelium of the common ancestor of all vertebrates [24].

In the rat VNO, Trpc2 is prominently and selectively localised to the microvilli of the receptor cells, which are thought to be the binding site of pheromones [12,25]. $\operatorname{Trpc} 2$ is expressed strongly in the VNO and in only a very small population of cells within the main olfactory epithelium (MOE) [12]. Mouse Trpc2 is localised to the anterior part of the sperm head where it is thought to be involved in the acrosome reaction, as $\mathrm{Ca}^{2+}$ entry and the acrosome reaction are both blocked by an antibody to Trpc2 [26]. However, $\operatorname{Trp} c 2^{-1-}$ null mutant mice are fertile, so the importance of Trpc2 for the acrosome reaction is in doubt $[11,27]$. $\operatorname{Trp} c 2^{-1-}$ mice do, however, provide dramatic evidence for the importance of Trpc2 in VNO function [11,27]. Trpc2 $2^{-/-}$male mice fail to initiate attack behavior at the approach of an intruding male and fail to establish a rank order [11,27]. $\operatorname{Trpc} 2^{-1-}$ males also mount other males far more than normal males do. $T R P C 2^{-1-}$ null mutant mice show not only a change in behaviour [11,27], but also exhibit differential hypotrophy of the glomerular layer of the AOB, with the anterior portion of the glomerular layer (innervated by V1R cells) resembling that of wild-type mice, and the posterior portion (innervated by V2R cells) reduced or absent [28].

Vannier et al. 1999 [16] identified two transcripts of $m \operatorname{Trpc} 2$ ( $m$ TRPC2A and $m T R P C 2 B)$ that were exclusively expressed in testis, whereas a later study [29] failed to find these two transcripts but described two shorter transcripts called $m T R P C 2 \alpha$ and $m$ TRPC $2 \beta$. The predicted proteins encoded by $m T R P C 2 \alpha$ and $m$ TRPC $2 \beta$ lacked the N-termini of mTRPC2A and mTRPC2B and resembled more rat Trpc 2 and other TRPC proteins. $m T R P C 2 \beta$ was found to be strongly expressed in the VNO and only weakly expressed in testis, suggesting it may represent the more biologically relevant variant.

As TRPC2 is relatively conserved among species (e.g. [24]) in contrast to $V 1 R$ or $V 2 R$ genes (e.g. [6-10]), it is more suited for investigating the evolution of the VNR pathway in vertebrates and especially in mammals. To date, TRPC2 has only been studied in eutherian mammals but not in marsupials or monotremes. Further data may reveal whether a longer transcript also exists in species other than mouse or whether the shorter transcript mTRPC2 $\beta$ is more similar to other species as described in rat. Using genome sequence data from two divergent marsupials, the tammar wallaby (Macropus eugenii) and the gray short-tailed opossum (Monodelphis domestica), we analysed the TRPC2 locus of marsupials and compared it to that of other vertebrates. We also include an analysis of this locus in another divergent mammal, the platypus (Ornithorhyncus anatinus). By a combination of cDNA cloning, expression analysis and bioinformatics, we show that the locus orthologous to mouse Trpc2 is comprised of two distinct genes that are regulated independently.

\section{Results}

\section{The TRPC2 locus is comprised of two independently regulated genes}

In order to investigate the presence of TRPC2 orthologues in marsupials, we performed BLAST searches of genomic databases of the grey short-tailed opossum and the tammar. The longest murine $\operatorname{Trpc} 2$ transcript [GenBank: NM_011644] contains 23 exons, with the coding sequence spanning Exons 2-23 (Figure 1). Sequences orthologous to all murine coding exons were identified in the genomes of both tammar and opossum. For both species, splice donor and acceptors sites were identified for all coding exons with the exception of the 3' donor site of Exon 10 and the 5 ' acceptor site of Exon 11. Retention of the intron between Exons 10 and 11 was predicted to result in a truncated protein. Nevertheless, Exons 12-23 of both marsupial species had an uninterrupted open reading frame (ORF).

To elucidate the splicing arrangement of TRPC2 transcripts in the tammar, RT-PCR was performed on adult female VNO-derived cDNA. RT-PCR using primers specific to Exons $2(1 \mathrm{~F})$ and 23 (1R) produced two faint bands of slightly differing size (not shown). This PCR was used as a template for several nested PCRs to amplify fragments of TRPC2 CDNA. Using nested primers specific to Exons $2(2 \mathrm{~F})$ and $23(2 \mathrm{R})$, one fragment was cloned that was sequenced and found to represent Exons 2-9 and 13-23 in contiguity (see predicted tammar transcript A (Figure 1)). PCR using nested primers specific to Exons $2(2 \mathrm{~F})$ and $14(5 \mathrm{R})$ yielded two more cloned products of differing size. Sequencing of the first of these showed it to represent Exons 2-9 and 12-14 in contiguity (see predicted tammar transcript B (Figure 1)). Sequencing of the second clone showed it to be similar to the first except that Exon 6 was longer (resulting from the use of an alternate splice donor site) and that Exon 12 was omitted (see predicted tammar transcript $\mathrm{C}$ (Figure 1)).

The predicted ORFs of all three of the above clones would result in substantially truncated proteins. Because we were unable to amplify any products containing putative Exons 10 and/or 11, an additional RT-PCR was 


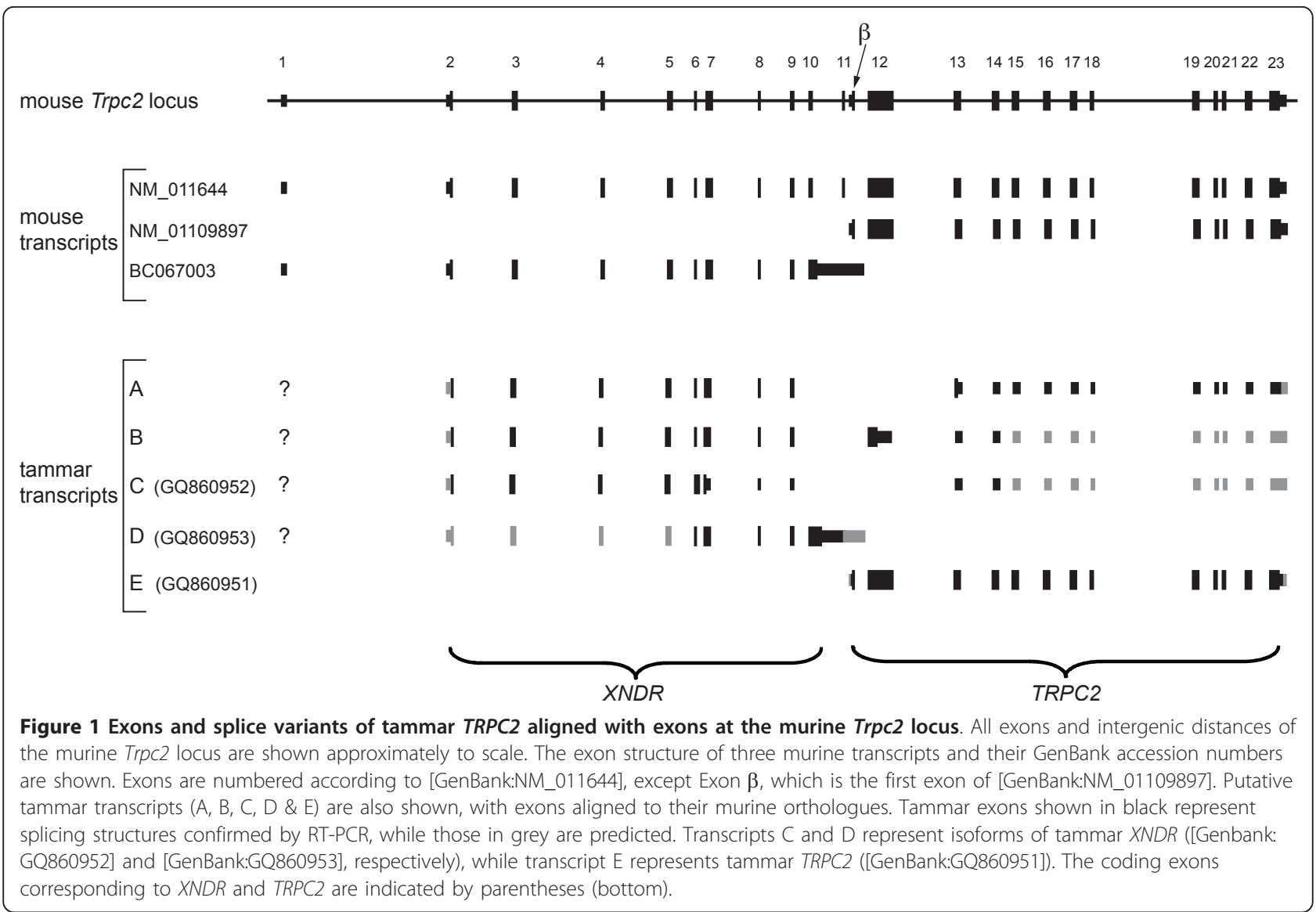

performed using primers specific to Exon $6(3 \mathrm{~F})$ and the 3' part of Exon 11 (6R). This resulted in a cloned fragment representing Exons 6-11 but including intronic sequence between Exons 10 and 11 (see predicted tammar transcript D (Figure 1)). Sequence from this clone also encoded a predicted truncated protein. From our RT-PCR results and the apparent absence of appropriate splice sites, we conclude that in marsupials no intron is ever spliced out between the regions orthologous to murine Exons 10 and 11.

The conservation of predicted amino acid sequence from both Exons 2-9 and 12-23 suggested that both regions are functional but do not encode the same protein. Evidence from previous reports [12,29] suggested that a distinct promoter downstream of Exons 2-9 drives expression of Exons 12-23 specifically in the VNO. In fact, in no species other than the mouse could we find any evidence for a transcript with a continuous ORF that included both regions. We therefore compared expression by non-nested RT-PCR using primer pairs specific to each region. Primers specific to Exons $6(3 \mathrm{~F})$ and 7 (4R) yielded two products (resulting from each of the alternate splice donor sites of Exon 6 (above)) from a broad range of adult tissues, whereas primers specific to
Exons 12 (4F) and 14 (5R) yielded a product only from adult VNO (Figure 2A).

We next examined the onset of VNO-specific expression (Exons 12-14) in early tammar pouch young. Because of the difficulty in separating VNO from main olfactory epithelium (MOE) and other tissues in the nasal region of the smallest pouch young (the neonate weighs only $400 \mathrm{mg}$ ), we also tested markers of sensory neurons (SOX2 [30]) and MOE (CNGA2 (also called oCNC1) [31]). RT-PCR product for Exons 12-14 was detected strongly in the VNO of day 34 and day 52 pouch young, very weakly in some earlier pouch young VNO and MOE samples and very weakly in adult liver and lung (Figure 2B). Detection of CNGA2 in both VNO and MOE samples of some early pouch young suggested that there may have been cross-contamination of these tissues or that VNO- and MOE-specific receptor neurons have not fully differentiated from each other at these very early stages of development.

Our results indicated that a second, VNO-specific promoter lies upstream of tammar Exon 12, as previously postulated for the mouse [32]. We found that the putative first exon-"Exon $\beta$ "-of a mouse VNO-specific transcript [32] is also conserved in sequence upstream of Exon 12 in 


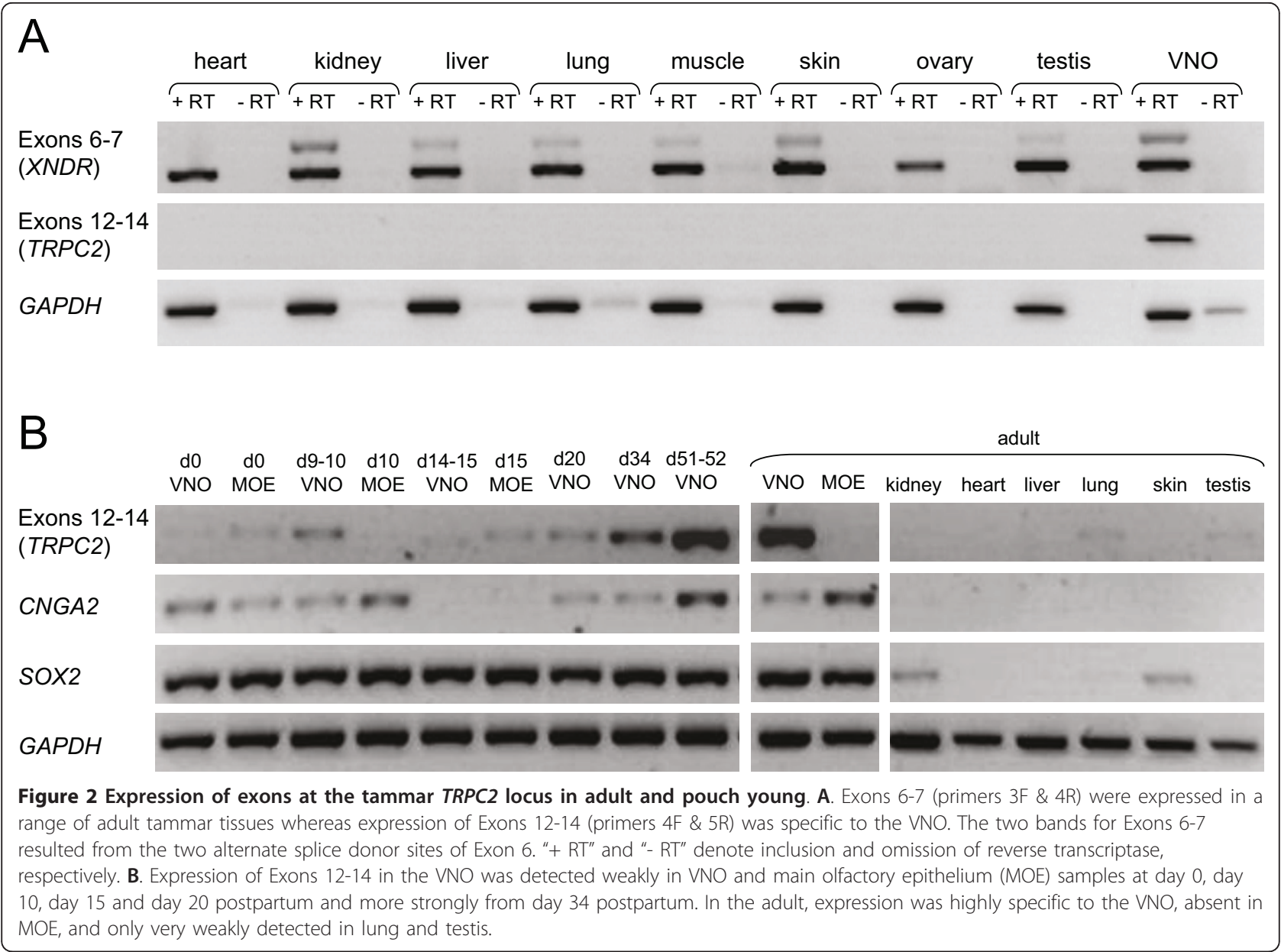

the tammar wallaby as well as many other mammal species (Figure 3). RT-PCR of tammar VNO-derived cDNA using primers specific to Exons $\beta$ (5F) and 23 (3R) revealed a full-length open reading frame, represented by transcript E (Figure 1) [GenBank:GQ860951].

The essential domains for TRPC2 function, including the transmembrane and cytoplasmic domains, are encoded by Exons $\beta$-23. Exons 2-9 were predicted to encode a protein with similarities to the $\mathrm{N}$-terminal domain of XRCC1 (X-ray repair complementing defective repair in Chinese hamster cells 1). Our expression and bioinformatic data indicate that the latter exons comprise a gene distinct from TRPC2, which we term $X N D R$ (Xrcc1 N-terminal domain related). Murine $X n d r$ is therefore represented by murine transcript [GenBank:BC067003] (Figure 1). Hence we refer to transcripts comprised of Exons $\beta-23$ as representing TRPC2 (corresponding to murine transcript [GenBank:NM_001109897] (Figure 1)) and transcripts encompassing both regions as representing XNDR-TRPC2 (corresponding to murine transcript [GenBank:NM 011644] (Figure 1)). In the mouse, Xndr-Trpc2 (previously called isoform 1 of $\operatorname{Trpc} 2$ ) is thus encoded by transcripts initiated by the $X n d r$ promoter in which splicing occurs between the donor site of Exon 10 and the acceptor site of Exon 11, whereas these splice sites are not used in transcripts that encode Xndr.

\section{XNDR and TRPC2 are both highly conserved among vertebrates}

We examined the evolution of the XNDR/TRPC2 locus by comparing the assembled genomes of a broad range of vertebrate species available on the UCSC browser. Orthologues of $X N D R$ appear to be present in all jawed vertebrates ranging from teleost fishes to mammals (Figure 4). However, we were unable to detect orthologues of $X N D R$ in lower deuterostomes such as lancelet and sea squirt, in which the closest homologues appear to represent orthologues of XRCC1 (data not shown). In human and chimpanzee (Pan troglodytes), the gene is disrupted, with Exons 1-5 located approximately $68 \mathrm{Mbp}$ distant to Exons 6-10 on chromosome 11 of both species. This is a relatively recent event, however, as all 10 exons are normally arranged in another hominid, the orangutan, as well as in other primates, with no disruption of the ORF 
mouse CCGTTCCCCTT.GTCCCCAAGGTAC......................

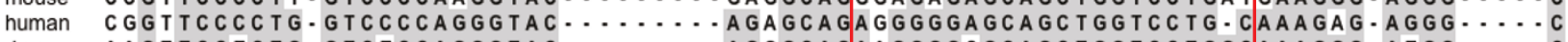

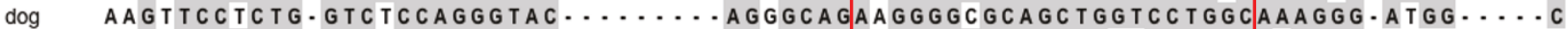

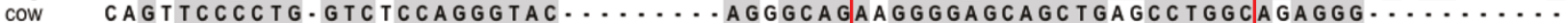
elephant CAGCTCC C C TG. GTCCCCAGGGTAC.............. tammar TTGCT-CTCTGGGTCCCTGAGGGGCCAAGCTGGGATAAAGGATGGGGAGCAGCTGGTCCTGGCAGGGGGCAGGGCC CAGC opossum TTGCT - C TC T GCATTC C CAAGGGGCAACGCTGGGGCAGAGGATGGGGAGCAGCTGGTCCTGGCAGGGGGCAGGGCC CAGC platypus GTGCCCACCCCTCGGCCCAGGGGA.ACCGATG.CGGAGGTAGAGGGGAGGCCCCCG..TGG...GG. TGGC.ACCC

\section{$\mathrm{GC}$ box} $\checkmark$ transcription start site

mouse ACAGGGTGGGGCTTTGGG.T. . AGAAATAAAAGAGAATGCTAGGCACAGTCAAGGGAG....AGGCAG...AGCTGGA human CCAGGTCAGAGTTTTGAA. T...AGATAGAAAAGAGGGTGCTAGGCACAGTCA.GGGAG...AAGACTG...AGCAGGA $\operatorname{dog}$ TCAGGGCGGGGTTTTGAACTCAAGGGTAGGAAAGCGGAAGCTGGGCACAGTCAGTGGAGCCAAGGAGCAGGAGAGCAGGA cow AAGGGCAGGTTTGGAAG.... GGGTAGGAAAGAGAAAGCTGGGCACAGCTAGGGGAGC...AGACAGAGGAGCCGGA elephant CCAGGGTGGGGTTTT.AAGT. . GGGTAGGATAGAGGATGCCAGGCACCATCAGGGGAGC...AGAC....AGCAGGA tammar CTAGGGCGGGGTCT.......... GGGTGCATAAGAAAGAATTGGACATCCCAGAGAGAGC....AAACAG...AGCAAGA opossum CTAGGGCGGGGTCT. ........ GGGTGCA TAAGAGAGCGA TGGGTA TCC CAAAGGGAGA... AAACAAT . AAGAAGA platypus CGAGGGCGGAG.C.GCGGCC...GGGAGGATC.GT.CGGGAGGGGCCGGGCGCACTCAGACGGGAGACGG...CACAGGG

mouse GTTCAAGCATTCAGCCCC.......... TGCCCACAGAGGTGGGTGCAGCCATGGACCCCCTTTCGGTAAGAGACCCC human GTTCAGTCCTTCGGTTCCTGTCAGCACAGGTGCCAGCATAGGTGGGCACAGCCATGGACCCCATCACGGTAAGAAGCCTG dog GTTAAATCTTTCGCTCCC........... TACCAGCATAAGTGGGTGCAGCCATGGACCTCCTCACGGTAAGAGGCCTG cow GTTCTCTCCTGCCATCCC.................. elephant GTTCAGTCCTTTGATCTG........... TGCCAGCGTAGGTGGGTGCAGCCATGGACCCCCTCAAGGTAAGAGGCCTT

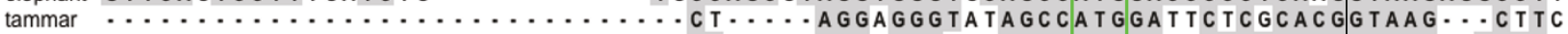

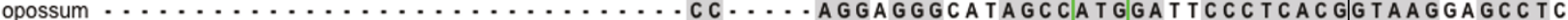
platypus AACGGAGCCCCCATCAGC.GTCGGC.GGGCTCAGGGCCTCTTCCAGCTCGGCCATGGACTCCAACTCGGTAAGA. GCCTG start codon

Figure 3 Alignment of the putative promoter and Exon $\beta$ of TRPC2 from selected mammal species. High sequence conservation is observed near the boundary between Exon $\beta$ and the adjacent intron, as well as at a putative HEN1 binding site and GC box. The indicated transcription start site corresponds to that of the mouse $\operatorname{Trpc} 2 \beta$ transcript.

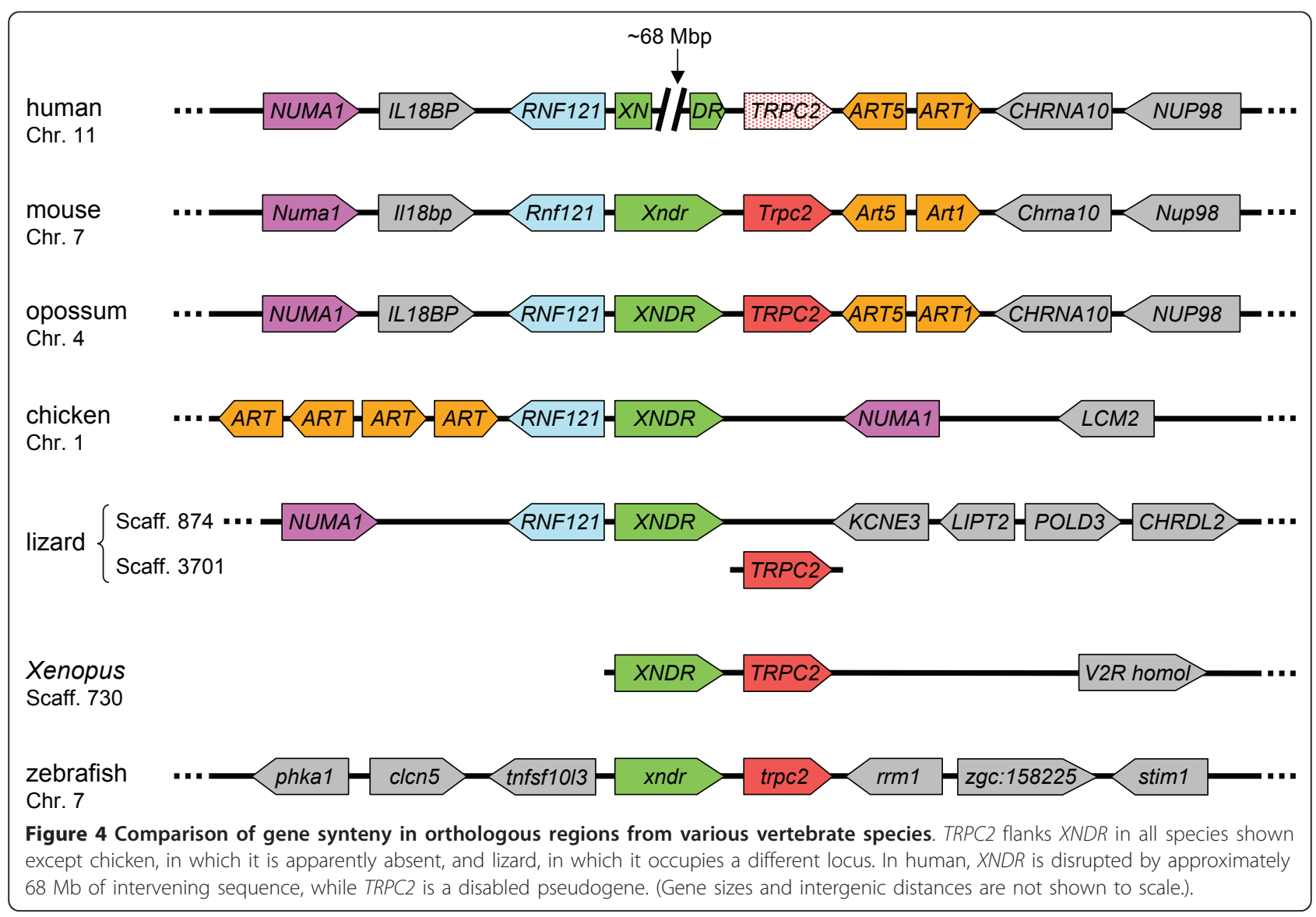


(not shown). The N-terminal region of XNDR encoded by Exons 2-5 is the more highly conserved (data not shown) and presumably the more functionally important. Thus the non-disrupted ORF of Exons 2-5 may still be functional in both human and chimpanzee.

TRPC2 orthologues in human and other catarrhine primates have a disrupted ORF, as previously reported $[17,19,23]$. In the chimpanzee, Exons 12-19 appear to have been completely lost, while in the orangutan (Pongo pygmaeus), a region corresponding to $\sim 4.75 \mathrm{~kb}$ in human and containing Exons 16-18 is deleted. In the Rhesus macaque (Macaca mulatta), all exons are present but Exon 16 is inverted (data not shown).

$X N D R$ and TRPC2 flank each other in all species in which the relative positions of both genes could be determined, with the exception of the anole lizard (Anolis carolinensis) in which they occupy separate genomic scaffolds (Figure 4). The close proximity of XNDR and TRPC2 appears to be ancestral, as it is also found in teleost fishes (Figure 4). There is also a second homologue of TRPC2 [GenBank:XM_683311] located on chromosome 15 of the zebrafish genome that does not appear to be present in other teleost species.

$X N D R$ and TRPC2 are differentially regulated in the platypus To confirm that independent transcriptional regulation of TRPC 2 and XNDR is conserved at least among mammals, we examined their expression in the platypus, one of the five living species of the third extant sub-order of mammals. Sequences orthologous to Exons 2-10, representing the complete coding sequence of $X N D R$, were identified in the platypus genome, along with Exons $\beta$, Exons 13-14, Exons 17-20 and part of Exon 23 of TRPC2 (data not shown). Although the platypus genome build is incomplete in this region, continuous sequence separates Exons 10 and $\beta$, showing that TRPC2 and XNDR flank each other. Forward primers designed within Exons 9 and $\beta$ and reverse primers designed within Exons 10 and 13 (Table 1) were used to test expression in adult tissues, including nasal tissue believed to contain the VNO, by RT-PCR. Platypus XNDR (Exons 9-10; 440-bp product) was detected in all tissues tested, whereas TRPC2 (Exons $\beta$-13; 881-bp product) was only detected in nasal tissue and liver (Figure 5). The sequence of the latter gel-extracted product has been deposited in GenBank [GenBank:HQ113234]. RT-PCR using the Exon 9 forward primer and the Exon 13 reverse primer did not yield any detectable product (not shown), suggesting that expression of an XNDR-TRPC2 transcript is low or absent in the platypus.

\section{The therian TRPC2 promoter contains a putative NHLH1 transcription factor binding site}

To characterise the promoter that may be responsible for specific expression of TRPC2 in the vomeronasal organ, we compared genomic sequence immediately upstream of the first exon $(\beta)$ of TRPC2 from a range of vertebrate species. When we compared those of mammals, two sites were identified as likely candidates: a HEN1 site and a GC box (Figure 3). The former shows strong similarity to the consensus binding site for NHLH1 (also called HEN1 or NSCL1), a neural-specific basic helix-loop-helix (bHLH) transcription factor [33]. The HEN1 site was well conserved among eutherians and marsupials, but not in the platypus (Figure 3) or in other vertebrates (not shown).

\section{Discussion}

$\operatorname{Trpc} 2$ was originally identified as encoding a VNO neuron-specific ion channel in the rat [12]. This transcript [GenBank:AF136401] corresponds to the transcript that we define here as TRPC2 proper. Two subsequent reports, however, identified additional variants of murine "Trpc2" transcript variants that were termed $\operatorname{Trpc} 2 A$, $\operatorname{Trpc} 2 B$ [16], $\operatorname{Tr} c p c 2 \alpha$ and $\operatorname{Trp} 22 \beta$ [29]. $\operatorname{Tr} p c 2 \beta$ was isolated by RACE-PCR from vomeronasal tissue and corresponds to the original rat $\operatorname{Trp} 22$ transcript. Each of the other transcripts included exons from $X n d r$ and, notably, was amplified from non-VNO-derived cDNA. The primers and methods used would have specifically favoured amplification of cDNA fragments of Xndr-Trpc2, rather than $X n d r$ or $\operatorname{Trpc2}$, even if they were at low abundance. Although Hofmann et al [29] reported that only $\operatorname{Trpc} 2 \beta$ was specifically expressed in the VNO and that two independent promoters are likely to be functional, until now there has been no formal recognition that $X n d r-\operatorname{Trp} c 2$ represents a fusion of two distinct genes and may be biologically irrelevant. At the time of preparing this manuscript, exons from both genes are still presented as defining "Trpc2" by the Entrez Gene database on the NCBI website.

In this study, we have presented several pieces of evidence to support the recognition of two distinct genes$X N D R$ and TRPC2 - at this locus in mammals. First, splice sites necessary for the full-length ORF of mouse $X n d r-T r p c 2$ are not conserved in most other species, rendering it implausible that XNDR-TRPC2 has any conserved role among vertebrates. Second, comparisons by RT-PCR using primers specific to either XNDR or $T R P C 2$ in the tammar wallaby and the platypus clearly supported the original evidence [29] that two independent promoters respectively regulate widespread (XNDR) and VNO-specific (TRPC2) expression. Third, analysis of a range of vertebrate genomes shows that the two genes have evolved independently. In the anole lizard, XNDR and TRPC 2 are both conserved but occupy separate genomic scaffolds. In the chicken, $X N D R$ is conserved whereas TRPC2 appears to be lost, or at least does not flank XNDR. In catarrhine primates, at least the first 4 
Table 1 Sequences of tammar-specific primers

\begin{tabular}{|c|c|c|c|}
\hline gene & name & sequence & location of primer binding \\
\hline XNDR- & $1 \mathrm{~F}$ & GGCTTATGGCTGCTCTCTTTCTGTGAC & Exon 2 \\
\hline \multirow[t]{10}{*}{ TRPC2 } & $2 \mathrm{~F}$ & TCCTCATGGCTCCTGTGAAGATCAG & Exon 2 \\
\hline & $3 \mathrm{~F}$ & CGGAGAACATTCTTCCCAGA & Exon 6 \\
\hline & $4 \mathrm{~F}$ & TTCCСTCTCCCGAATTAACA & Exon 12 \\
\hline & $5 F$ & AGCAAACAGAGCAAGACTAGGAGGGTATAG & Exon $\beta$ \\
\hline & $1 \mathrm{R}$ & TTCTTCCTGAAACCCCCTGTGAATC & Exon 23 \\
\hline & $2 R$ & TGAATCTCCACTTGGGCAGCGG & Exon 23 \\
\hline & $3 R$ & ATAGGTGGCACGGGGTTAGGTCAG & Exon 23 \\
\hline & $4 \mathrm{R}$ & ACATCTGAGCTGGCTGTGTG & Exon 7 \\
\hline & $5 R$ & GCCAAGTTTCCACACCAGAT & Exon 14 \\
\hline & $6 \mathrm{R}$ & TTGGGTTGGGGCTGGAATAGTAATG & Exon 11 \\
\hline \multirow[t]{2}{*}{$\overline{C N G A 2}$} & for & $\begin{array}{l}\text { TGTGCTTGATCCTGCTGGAGACTG } \\
\text {. }\end{array}$ & \\
\hline & rev & ACCACATCAGTGGGGATGACGG & \\
\hline \multirow[t]{2}{*}{$50 \times 2$} & for & TGGAGCAACGGCGGCTACGG & \\
\hline & rev & CCTGGAGTGCGACGACGAGG & \\
\hline \multirow[t]{2}{*}{ GAPDH } & for & CCTACTCCCAATGTATCTGTTGTGG & \\
\hline & rev & GGTGGAACTCCTITITTGACTGG & \\
\hline
\end{tabular}

coding exons of $X N D R$ are conserved and presumably functional, despite the loss of functionality of TRPC2. Together, our data provide overwhelming evidence for identifying $X N D R$ as a novel gene distinct from TRPC2. $X N D R$ encodes a predicted protein with similarities to the N-terminal domain of XRCC1 (X-ray repair complementing defective repair in Chinese hamster cells 1 ). XRCC1 has a key role in DNA base excision repair (reviewed [34]); thus it is possible XNDR has a similar role.

TRPC2 expression was highly specific to the VNO in the tammar, being highly expressed not only in the adult, but also in pouch young as early as day 34 post partum. Expression was only weakly detected at earlier stages, possibly due to the small proportion of expressing cells. Marsupials deliver highly altricial young that are equivalent to the eutherian fetus and complete most of their development post partum [35]. The surrounding tissue that was also dissected along with the VNO at these earlier stages may have diluted the proportion of TRPC2 transcripts in our samples. At birth, Goo protein

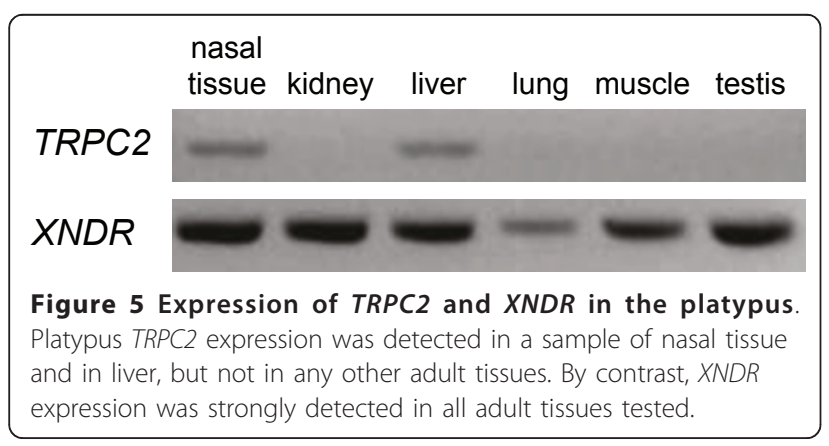

was confined to only a limited number of cells [36]. It thus seems likely that the VNO does not begin to function until around day 30 post partum in the tammar. This would make the main olfactory system the primary candidate for perception of pouch odours that guide the neonatal tammar into the pouch at birth [36]. Further studies will be needed to confirm whether the VNO is functional earlier in development in these highly altricial young.

We identified NHLH1 as a candidate regulator of TRPC2 expression. Mouse Nhlh1 is expressed specifically in the developing nervous system, peaking around the period of E10 to 12.5, and marks early post-mitotic neuroblasts $[37,38]$. Significantly, expression of NHLH1 becomes restricted during later stages to olfactory and vomeronasal receptor neurons [39]. Mammalian NHLH1 and NHLH2 are encoded by paralogous genes and are almost identical in their $\mathrm{C}$-terminal bHLH domain but highly divergent in their N-terminal domain [40]. Murine Nhlh 2 is also expressed in the developing nervous system, peaking at around E11.5 [40]. In the NCBI UniGene gene expression profile estimated by expressed sequence tag (EST) frequency in adult tissues, expression of Nhlh1, but not Nhlh2, is highly restricted to the nasopharynx http://www.ncbi.nlm.nih.gov/UniGene/ESTProfileViewer. cgi?uglist $=\mathrm{Mm} .2474$, consistent with a specific role for NHLH1 in mammalian olfactory and vomeronasal receptor neurons. We therefore propose that NHLH1 specifically regulates TRPC2 expression in VNO receptor neurons in mammals. We also found conserved E-box consensus sequences in the TRPC2 promoter of some teleost fishes (not shown), suggesting that other bHLH transcription factors may be involved in regulating 
TRPC2 in other species. The genomes of teleost fishes only appear to contain an orthologue of NHLH2 but not NHLH1, suggesting that $N H L H 2$ represents the ancestral gene. Both jawed and jawless fishes only have a single olfactory organ, but the genetic components of a vomeronasal sensory system are nevertheless present in both groups [24]. It is possible that the evolution of NHLH1 is linked to the evolution of a morphologically more complex olfactory system in the tetrapod lineage.

\section{Conclusions}

By a combination of expression analysis, genomic analysis and a critical assessment of previous literature, our study clearly demonstrates that the locus formerly defined as encompassing a single gene in reality comprises two distinct genes: $X N D R$ and TRPC2. This distinction is important for future studies, especially for those comparing VNO function among divergent vertebrate species. $X N D R$ is broadly expressed and has a possible role in DNA repair, while TRPC2 is specifically expressed in the VNO under the probable regulation of NHLH1. The expression profile of TRPC2 in the tammar wallaby suggests that there is no TRPC2-dependent role for the VNO during early pouch life.

\section{Methods}

\section{Animals}

Tammar wallabies were obtained from our breeding colony held under permits from the Victorian Department of Natural Resources and Environment. Platypus tissues were collected from two adult males trapped in the Murrumbidgee River, NSW, under a permit from NSW National Parks \& Wildlife Service. Adult animals (tammar and platypus) were euthenised by an overdose of sodium pentobarbitone. Tammar pouch young younger than 40 days $(<1 \mathrm{~g})$ (that are heterothermic [35]) were cooled then decapitated. All experiments were approved by the University of Melbourne Animal Experimentation Ethics Committees and all animal handling and husbandry were in accordance with the National Health and Medical Research Council of Australia (2004) guidelines.

\section{Tissues}

Heart, kidney, liver, lung, muscle, skin, ovary, testis, retina, tongue (from the tip), MOE and VNO tissue were collected from adult female tammar wallabies. VNO tissue was collected from pouch young of age 0 , $10,15,20,34$ and 52 days post partum (each $\mathrm{n}=1$ ). MOE was collected from pouch young of age 0,10 and 15 days post partum. As the structure of the VNO and $\mathrm{MOE}$ are not visible under the dissecting microscope at the age of 0 to 15 days, the areas in which they lie were dissected out. All samples were snap frozen with liquid nitrogen and stored at $-80^{\circ} \mathrm{C}$ until use.

\section{RNA extraction and RT-PCR}

RNA was extracted using the RNeasy Lipid Tissue Mini kit (QIAGEN, Doncaster Victoria, Australia; \#74804) for $\mathrm{VNO}$ and brain tissue and TRI-reagent for the other tissues. RNA was DNase-treated (Ambion, USA; \#AM1906). For each sample, $8 \mu \mathrm{g}$ was reverse transcribed using SuperScript ${ }^{\mathrm{TM} I I I}$ (Invitrogen; Mount Waverley, Victoria, Australia; \#12574-018) in a total reaction volume of $20 \mu \mathrm{L}$.

For cloning and sequencing of cDNA fragments, an initial PCR was performed using primers (1F and $1 \mathrm{R}$ ) spanning Exons 2-23 (Table 1). PCR was performed using ExTaq Polymerase (TaKaRa) according to the manufacturer's instructions, in a $20 \mu \mathrm{L}$ volume containing $0.5 \mu \mathrm{L}$ of 1st strand cDNA template as follows: $95^{\circ} \mathrm{C}$ for $1 \mathrm{~min}$.; 40 cycles of $95^{\circ} \mathrm{C}$ for $20 \mathrm{sec}, 60^{\circ} \mathrm{C}$ for 20 sec., $68^{\circ} \mathrm{C}$ for 4 min.; $68^{\circ} \mathrm{C}$ for $1 \mathrm{~min}$. Nested PCRs were performed using identical conditions to above except that different primers were used (Table 1) and the template was $0.5 \mu \mathrm{L}$ of the initial PCR. RT-PCR products were cloned to PGEM-TEasy (Promega, NSW, Australia) for sequencing.

For tammar expression studies, PCR was performed using GoTaq Green (Promega, NSW, Australia) according to the manufacturer's instructions, in a $10-\mu \mathrm{L}$ volume containing $0.5 \mu \mathrm{L}$ of 1 st strand cDNA template as follows: $95^{\circ} \mathrm{C}$ for 1 min.; 35 cycles of $95^{\circ} \mathrm{C}$ for $20 \mathrm{sec}, 60^{\circ} \mathrm{C}$ for $20 \mathrm{sec}$., $72^{\circ} \mathrm{C}$ for $1 \mathrm{~min}$.; $72^{\circ} \mathrm{C}$ for $1 \mathrm{~min}$. For platypus expression studies, PCR conditions were identical except that ExTaq Polymerase was used according to the manufacturer's instructions and the annealing temperature was $68^{\circ} \mathrm{C}$. Primer sequences were as indicated in Tables 1 and 2 .

\section{Sequence analysis}

NCBI BLAST http://blast.ncbi.nlm.nih.gov/Blast.cgi was used to search whole genome shotgun (WGS) databases and trace archives. The UCSC browser http://www.genome.ucsc.edu/ was used to search and analyse assembled genomes. Contigs were assembled from trace archive sequences using CAP3 http://pbil.univ-lyon1.fr/cap3.php. Identification of exons was aided by use of GENSCAN http://genes.mit.edu/GENSCAN.html. Sequence alignments were performed using ClustalW in the MacVector software package. Tammar sequences assembled from cloned cDNA fragments were deposited in GenBank as

$\begin{aligned} & \text { Table } 2 \text { Sequences of platypus XNDR-TRPC2-specific } \\
& \text { primers }\end{aligned}$
\begin{tabular}{llll}
\hline Gene & name & sequence & $\begin{array}{l}\text { location of } \\
\text { primer binding }\end{array}$ \\
\hline XNDR & p1F & GCAGAGGACGGAGAGATGGGTCAAG & Exon 9 \\
& p1R & CGAGGAGGGGAGGAGTGGAAC & Exon $\beta$ \\
\hline$T R P C 2$ & p2F & GGAGACGGCACAGGGAACGG & Exon 10 \\
& p2R & TTGAGCACGGCGGTCACCTC & Exon 13 \\
\hline
\end{tabular}


follows: TRPC2 [GenBank:GQ860951], XNDR isoform 1 [GenBank:GQ860953], XNDR isoform 2 [GenBank: GQ860953]. Sequences for promoter analysis were taken from genome builds on the UCSC browser for all species, except tammar wallaby, as follows: mouse build $m m 9$, chr7:109231513-109231712; human build $h g 19$, chr11:36 36855-3636875; dog build canFam2, chr21:2918868929188900; cow build bosTau4, chr15:51030125-51029929; elephant build loxAfr3, scaffold_79:7861327-7861525; opossum build monDom5, chr4:348474319-348474426; platypus build ornAna1, Contig17150:12022-12235; and tammar wallaby [GenBank:ABQO011015996], 388-206.

\section{Acknowledgements}

We thank Kerry Martin and Scott Brownlees, for assistance with animal handling, and Andrew Pask and Melissa Gamat for their helpful suggestions concerning the investigations of tammar TRPC2. This work was supported by a grant from The Holsworth Wildlife Research Endowment (to NYS) and by an Australian Research Council Federation Fellowship (to MBR).

\section{Authors' contributions}

The experiments were conducted and evaluated by SF and NYS. NYS, MBR, TF and GS assisted in the collection of tissues. SF, NYS and MBR prepared the manuscript. All authors discussed the results and commented on the manuscript. All authors read and approved the final manuscript.

Received: 7 April 2010 Accepted: 19 August 2011 Published: 19 August 2011

\section{References}

1. Halpern M: The organization and function of the vomeronasal system. Annu Rev Neurosci 1987, 10:325-362.

2. Halpern M, Martinez-Marcos A: Structure and function of the vomeronasal system: an update. Progress in Neurobiology 2003, 70:245-318.

3. Johnston RE: Chemical communication and pheromones: the types of chemical signals and the role of the vomeronasal system. In The neurobiology of taste and smell.. 2 edition. Edited by: Finger TE, Silver WL, Restrepo D. New York: Wiley-Liss; 2000:99-125.

4. Sam M, Vora S, Malnic B, Ma W, Novotny MV, Buck LB: Neuropharmacology. Odorants may arouse instinctive behaviours. Nature 2001, 412:142

5. Trinh K, Storm DR: Vomeronasal organ detects odorants in absence of signalling through main olfactory epithelium. Nat Neurosci 2003, 6:519-525

6. Bjarnadóttir TK, Fredriksson R, Schiöth HB: The gene repertoire and the common evolutionary history of glutamate, pheromone (V2R), taste(1) and other related $G$ protein-coupled receptors. Gene 2005, 362:70-84.

7. Grus WE, Shi P, Zhang YP, Zhang J: Dramatic variation of the vomeronasal pheromone receptor gene repertoire among five orders of placental and marsupial mammals. Proc Natl Acad Sci USA 2005, 102:5767-5772.

8. Shi $P$, Zhang J: Comparative genomic analysis identifies an evolutionary shift of vomeronasal receptor gene repertoires in the vertebrate transition from water to land. Genome Res 2007, 17:166-174.

9. Young JM, Trask BJ: V2R gene families degenerated in primates, dog and cow, but expanded in opossum. Trends Genet 2007, 23:212-215.

10. Young JM, Massa HF, Hsu L, Trask BJ: Extreme variability among mammalian V1R gene families. Genome Res 2010, 20:10-18.

11. Leypold BG, Yu CR, Leinders-Zufall T, Kim MM, Zufall F, Axel R: Altered sexual and social behaviors in trp2 mutant mice. Proc Natl Acad Sci USA 2002, 99:6376-6381.

12. Liman ER, Corey DP, Dulac C: TRP2: a candidate transduction channel for mammalian pheromone sensory signaling. Proc Natl Acad Sci USA 1999, 96:5791-5796.

13. Ramsey IS, Delling M, Clapham DE: An introduction to TRP channels. Annu Rev Physiol 2006, 68:619-647.
14. Vazquez G, Wedel BJ, Aziz O, Trebak M, Putney JW Jr: The mammalian TRPC cation channels. Biochim Biophys Acta 2004, 1742:21-36.

15. Clapham DE: TRP channels as cellular sensors. Nature 2003, 426:517-524.

16. Vannier B, Peyton M, Boulay G, Brown D, Qin N, Jiang M, Zhu X, Birnbaumer L: Mouse trp2, the homologue of the human $\operatorname{trpc} 2$ pseudogene, encodes mTrp2, a store depletion-activated capacitative Ca2+ entry channel. Proc Natl Acad Sci USA 1999, 96:2060-2064.

17. Liman ER, Innan $H$ : Relaxed selective pressure on an essential component of pheromone transduction in primate evolution. Proc Natl Acad Sci USA 2003, 100:3328-3332

18. Webb DM, Cortes-Ortiz L, Zhang J: Genetic evidence for the coexistence of pheromone perception and full trichromatic vision in howler monkeys. Mol Biol Evol 2004, 21:697-704.

19. Zhang J, Webb DM: Evolutionary deterioration of the vomeronasal pheromone transduction pathway in catarrhine primates. Proc Natl Acad Sci USA 2003, 100:8337-8341.

20. Wissenbach $U$, Schroth $G$, Philipp S, Flockerzi V: Structure and mRNA expression of a bovine trp homologue related to mammalian trp2 transcripts. FEBS Lett 1998, 429:61-66.

21. Yu L, Jin W, Wang JX, Zhang $X$, Chen MM, Zhu ZH, Lee $H$, Lee $M$, Zhang YP: Characterization of TRPC2, an essential genetic component of VNS chemoreception, provides insights into the evolution of pheromonal olfaction in secondary-adapted marine mammals. Mol Biol Evol 2010, 27:1467-1477.

22. Sato $Y$, Miyasaka N, Yoshihara $Y$ : Mutually exclusive glomerular innervation by two distinct types of olfactory sensory neurons revealed in transgenic zebrafish. J Neurosci 2005, 25:4889-4897.

23. Wes PD, Chevesich J, Jeromin A, Rosenberg C, Stetten G, Montell C: TRPC1, a human homolog of a Drosophila store-operated channel. Proc Natl Acad Sci USA 1995, 92:9652-9656.

24. Grus WE, Zhang J: Origin of the genetic components of the vomeronasal system in the common ancestor of all extant vertebrates. Mol Biol Evol 2009, 26:407-419.

25. Menco BP, Carr VM, Ezeh PI, Liman ER, Yankova MP: Ultrastructural localization of G-proteins and the channel protein TRP2 to microvilli of rat vomeronasal receptor cells. J Comp Neurol 2001, 438:468-489.

26. Jungnickel MK, Marrero H, Birnbaumer L, Lemos JR, Florman HM: Trp2 regulates entry of $\mathrm{Ca} 2+$ into mouse sperm triggered by egg ZP3. Nat Cell Biol 2001, 3:499-502.

27. Stowers L, Holy TE, Meister M, Dulac C, Koentges G: Loss of sex discrimination and male-male aggression in mice deficient for TRP2. Science 2002, 295:1493-1500.

28. Hasen NS, Gammie SC: $\operatorname{Trpc} 2$ gene impacts on maternal aggression, accessory olfactory bulb anatomy and brain activity. Genes Brain Behav 2009, 8:639-649.

29. Hofmann T, Schaefer M, Schultz G, Gudermann T: Cloning, expression and subcellular localization of two novel splice variants of mouse transient receptor potential channel 2. Biochem J 2000, 351:115-122.

30. Tucker ES, Lehtinen MK, Maynard T, Zirlinger M, Dulac C, Rawson N, Pevny L, LaMantia AS: Proliferative and transcriptional identity of distinct classes of neural precursors in the mammalian olfactory epithelium. Development 137(15):2471-2481.

31. Berghard A, Buck LB, Liman ER: Evidence for distinct signaling mechanisms in two mammalian olfactory sense organs. Proceedings of the National Academy of Sciences of the United States of America 1996, 93:2365-2369.

32. Yildirim E, Birnbaumer L: TRPC2: molecular biology and functional importance. Handb Exp Pharmacol 2007, , 179: 53-75.

33. Brown L, Baer R: HEN1 encodes a 20-kilodalton phosphoprotein that binds an extended E-box motif as a homodimer. Mol Cell Biol 1994, 14:1245-1255.

34. Caldecott KW: XRCC1 and DNA strand break repair. DNA Repair (Amst) 2003, 2:955-969.

35. Renfree MB: Society for Reproductive Biology Founders' Lecture 2006-life in the pouch: womb with a view. Reprod Fertil Dev 2006, 18:721-734.

36. Schneider NY, Fletcher TP, Shaw G, Renfree MB: The olfactory system of the tammar wallaby is developed at birth and directs the neonate to its mother's pouch odours. Reproduction 2009, 138:849-857.

37. Begley CG, Lipkowitz S, Gobel V, Mahon KA, Bertness V, Green AR, Gough NM, Kirsch IR: Molecular characterization of NSCL, a gene 
encoding a helix-loop-helix protein expressed in the developing nervous system. Proc Natl Acad Sci USA 1992, 89:38-42.

38. Murdoch JN, Eddleston J, Leblond-Bourget N, Stanier P, Copp AJ: Sequence and expression analysis of Nhlh1: a basic helix-loop-helix gene implicated in neurogenesis. Dev Genet 1999, 24:165-177.

39. Suzuki Y, Tsuruga E, Yajima T, Takeda M: Expression of bHLH transcription factors NSCL1 and NSCL2 in the mouse olfactory system. Chem Senses 2003, 28:603-608.

40. Göbel V, Lipkowitz S, Kozak CA, Kirsch IR: NSCL-2: a basic domain helixloop-helix gene expressed in early neurogenesis. Cell Growth Differ 1992, 3:143-148.

doi:10.1186/1471-2199-12-39

Cite this article as: Frankenberg et al:: Identification of two distinct genes at the vertebrate TRPC2 locus and their characterisation in a marsupial and a monotreme. BMC Molecular Biology 2011 12:39.

\section{Submit your next manuscript to BioMed Central} and take full advantage of:

- Convenient online submission

- Thorough peer review

- No space constraints or color figure charges

- Immediate publication on acceptance

- Inclusion in PubMed, CAS, Scopus and Google Scholar

- Research which is freely available for redistribution

Submit your manuscript at www.biomedcentral.com/submit
C Biomed Central 